\title{
Querschnittsstudie
}

\section{Viele Geburtshelfer leiden unter posttraumatischem Stress}

\author{
Geburten sind ein riskantes Unternehmen, nicht nur für Mutter und Kind, sondern \\ auch für die betreuenden Ärzte und Hebammen. Nicht wenige brechen unter der Belastung \\ zusammen, wie die Ergebnisse einer schwedischen Studie verdeutlichen.
}

\begin{abstract}
_ Sterbende oder schwer geschädigte Neugeborene, tote oder schwer verletzte Mütter - in einer Befragung von gut 700 ärztlichen Geburtshelfern und knapp 1.400 Hebammen antworteten $84 \%$ der Ärzte und 71\% der Hebammen, so etwas schon mindestens einmal mitgemacht zu haben. $43 \%$ von ihnen berichteten, während der Ereignisse von Furcht, Hilflosigkeit oder Panik ergriffen worden zu sein. Oft sahen sie sich in ihrem beruflichen Rollenverständnis bedroht (25\% der Ärzte, 17\% der Hebammen). Noch häufiger wurden sie nach dem Trauma von Schuldgefühlen geplagt (47\% bzw. 28\%).
\end{abstract}

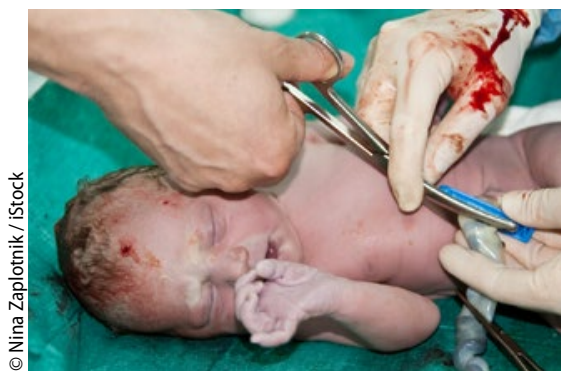

Die Geburt bedeutet Stress - nicht nur für Mutter und Kind.

In beiden Berufsgruppen betrug der Anteil von Personen, die Symptome einer partiellen posttraumatischen Belastungsstörung (Post-Traumatic Stress-
Disorder, PTSD) zeigten, 15\%. Und 7\% der Ärzte sowie 5\% der Hebammen erfüllten die Symptomkriterien einer voll ausgeprägten PTSD.

PTSD-Symptome hatten Konsequenzen für die Arbeit der Betroffenen. Häufig gaben sie die Arbeit im Kreißsaal auf (17\% der Hebammen). 13\% der Ärzte und $11 \%$ der Hebammen wechselten in eine ambulante Tätigkeit. Traumatisierte Hebammen wurden auch öfter krankgeschrieben als ihre nicht belasteten Kolleginnen (7\% vs. $1 \%$ ).

Dr. Robert Bublak

- Wahlberg Å et al. BJOG 2016, online 24. August; doi: 10.1111/1471-0528.14259

\section{Hier steht eine Anzeige.}

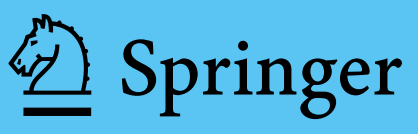

